Original Research Paper

\title{
Some Morphologic Features of Heart Failure with Different Etiology
}

\author{
Dmitrij Arkadievich Oleynikov \\ Department of Therapy, St. Petersburg State Academy of Veterinary Medicine, St. Petersburg, Russia
}

\author{
Article history \\ Received: 15-03-2017 \\ Revised: 13-05-2017 \\ Accepted: 20-05-2017 \\ Email: wolfberg.guard@gmail.com
}

\section{Introduction}

Doxorubicin is dose-depended cardio toxic chemotherapy agent. Due to its effective is wide spread in veterinary chemotherapy protocols, but its excessive use often leads to irreversible dilated cardiomyopathy phenotype disease (Swain et al., 2003; Olson and Mushlin, 1990). This cardiotoxicity is connected with metabolic alterations and morphological changes. Usually this disease has long latent period and in last stages manifest with biventricular insufficiency, heart failure and death (Billingham et al., 1978; Broder et al., 2008; Swain et al., 2003). Due to the fact that most of oncology patients are geriatric and usually already have some alterations in myocardial function we need to define morphological differences between most common heart diseases in canine. In this study we tried to differentiate features of heart failure developing due to Chronic Degenerative Valvular Disease (CDVD), Dilated Cardiomyopathy (DCM) and doxorubicininduced cardiomyopathy (DoxCM).

\section{Animals, Materials, Methods}

In this study, we studied 46 dogs of different sex, age and breed. All the dogs were patients of veterinary clinics "Pride" and "Champion", Saint Petersburg, Russia. Groups were formed due to primary diagnosis: (1) Control (animals without cardiology problems, $\mathrm{n}=14$; mixture of half-breed dogs); (2) CDVD (animals with chronic degenerative valvular disease, $\mathrm{n}=16$; 6- Yorkshire terrier, 4 - Toy Poodle, 4 - Toy Terrier, 2 - Chihuahua); (3) DCM (patients with idiopathic dilated cardiomyopathy and dilated cardiomyopathy-phenotype changes, $\mathrm{n}=6 ; 2-$ Rotveilers, 2 - Cane corso, 1 - Ca De Bou, 1 - Labrador Retriever); (4) DoxCM (dogs, treated with doxorubicin, $n$ $=10 ; 8$ - half-breed dogs, 2 - German shepherds).

The aim of this study is to elucidate some morphological aspects of each nosologic units. Myocardium for research needs was obtained immediately after euthanasia on autopsy.

Studied animals were diagnosed by standard methods (clinical findings, blood analyzes, radiography, echocardiography, electrocardiography). Patients in 
control groupdid not have diagnosed myocardial diseases and were euthanized due to low life quality. In other groups euthanasia were admitted in cases of end-stage and refractory heart failure and inability to sustain live functions or by owners will. Obtaining of samples were agreed with owners.

Patients with chronic heart failure had clinical signs of antero - and retrorgrade insufficiency, uncontrolled pulmonary edema and secondary pulmonary hypertension (CDVD group), syncope cases, lethargy; some dogs were euthanized during surgery due to severe neoplastic process or owners will. CDVD diagnose was also proved by autopsy changes in mitral valve, DCM by myocardial observation and comparison with healthy dogs. DoxCM - had in their history chemotherapy with doxorubicin and echocardiographic signs of myocardial morphological or functional changes.

Materials were obtained right after death. Samples were taken from left ventricular apex. This localization is preferred for comparative analyzing with DoxCM (Swain et al., 2013; Minotti et al., 2004). Material for histology fixed in neutral formalin and Bouin solution, for ultramicroscopic study - in glutaraldehyde.

Histology samples were standardly prepared and stained with hematoxylin and eosin, metal hematoxylin, picrofuchsin and by Mallory. Ultramicroscopy studies were done in standard technic.

\section{Results}

In stained with hematoxylin and eosin samples from control group we defined well-ordered myocardial fibers, unchanged interstitial compound. Cytoplasm has normal striation, myofibrils orientated parallel to outer membrane of cardiomyocyte. Nucleuses were prolonged, located centrally with 1-2 nucleolus, heterochromatin condensed in peripheral part of nucleus. Myocardial stroma has non-significant amounts of fibroblasts and collagen fibers. Microcirculatory was presented by venules, arterioles and capillary net. Venules and capillary has thin walls, lined by endotheliocytes, with non-significant amounts of blood cells in the lumen. In arterioles we observed myocytes, endothelial nucleus were orientated in parallel to basal membrane. In Mallory stained samples we found non-significant amounts of collagen fibers in interstitial spaces, vessels walls, epi- and endocardium. In addition, we stained myocardium with metal hematoxylin and picrofuchsin and defined well-ordered myofilaments localization, without significant spaces of non-specific cytoplasm.

Further, we studied samples from CDVD group. We observed separation and dissociation of myocardial fibers. Many of cardiomyocytes have 2 nucleuses. Contractile component mostly unchanged. There are sporadic fibroblast and adipocytes proliferation. These remodeling features are found mostly in subepicardial spaces (Fig. 1). In Mallory, metal hematoxylin and picrofuchsin stained samples we observed increased amounts of collagen fibers, adipose tissue proliferation, myofibrils were separated and had two or more nucleuses, also we admitted increased spaces of nonspecific cytoplasm (Fig. 2)

During examination of DoxCM samples, we defined significant changes in tissue and cell morphology. On micro photos, we can observe subendocardial part of left ventricular myocardium, what has deep degenerative changes, characterized replacement of contractile components with adipose tissue and myocardial cells separation. Contractile filaments in most cases are represented in cells, but significant amounts of cardiomyocytes myofibrils are replaced by non-specific cytoplasm, we also can observe lipofuscin inclusions, vacuoles (lipid droplets). In represented samples, we see many polynucleated cells, which is marker of decompensated condition of myocardium-polynucleated cardiomyocytes are terminally differentiated and can't be divided or compensatory hypertrophied. We defined specific localization of alteration signs in subendocardial parts of myocardium. This can be explained by the mechanism of Dox cardiotoxicity. Dox-induced metabolic disturbances are more likely to develop in sites with less availability to energy substrates and Dox $\mathrm{Ca}+2$ interaction determined contracture of myofilaments, endothelium inflammation can provide microcirculatory compression (Fig. 2). Dox-induced inflammatory of vessels is one of anthracycline cardiotoxicity worsen factors. Myocardial microcirculatory vessels and endotheliocytes are highly affected by this action. On micro photos, we can define intima and media hyperplasia, leucocytes migrating to inner wall and surrounding tissues (Fig. 3). On samples stained by Mallory we observe high amounts of collagen fibers, myocardiocytes separation, interstitial edema, vessels media hyperplasia, many apoptotic bodies (Fig. 3). Metal hematoxylin and picrofuchsin stained samples were characterized with degeneration of myofilaments, lipofuscin inclusions, nucleuses degeneration, connective and adipose tissue proliferation (Fig. 4).

In DCM group, we admitted proliferation and cardiomyocytes replacement by adipose tissue and fibrosis. Cardiomyocytes were thinned, had lack of contractile filaments and their disposition to peripheral sites of the cell; many polynucleated cell, but not as much as in DoxCM group.Mallory stained samples represents proliferation of connective tissue, massive fibrosis, interstitial edema, cardiomyocytes vacuolization (Fig. 5). Metal hematoxylin and picrofuchsin stained samples also showed massive fibrosis and adipose tissue proliferation. We admitted high amounts of collagen fibers and fibroblasts proliferation (Fig. 5). 


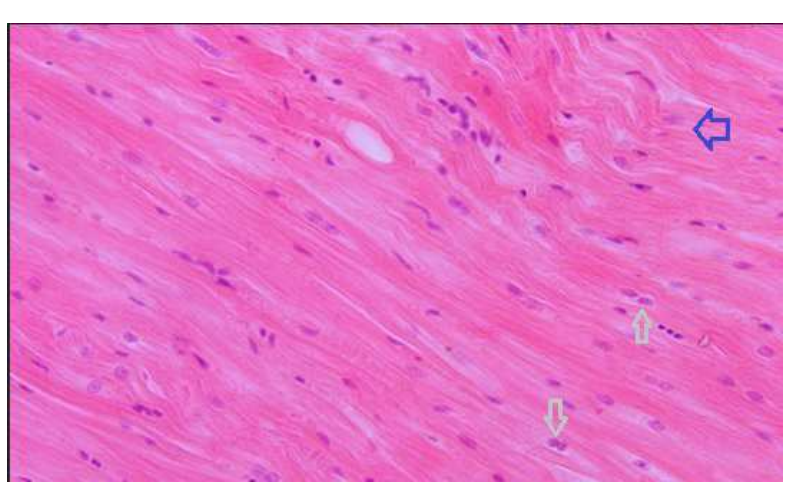

Fig. 1. CDVD group. Cardiomyocytes. Polynuclear cardiomyocytes (gray arrows). Cardiomyocyte degradation. Separation of fibers (blue arrow). Hematoxylin\&eosin, x200

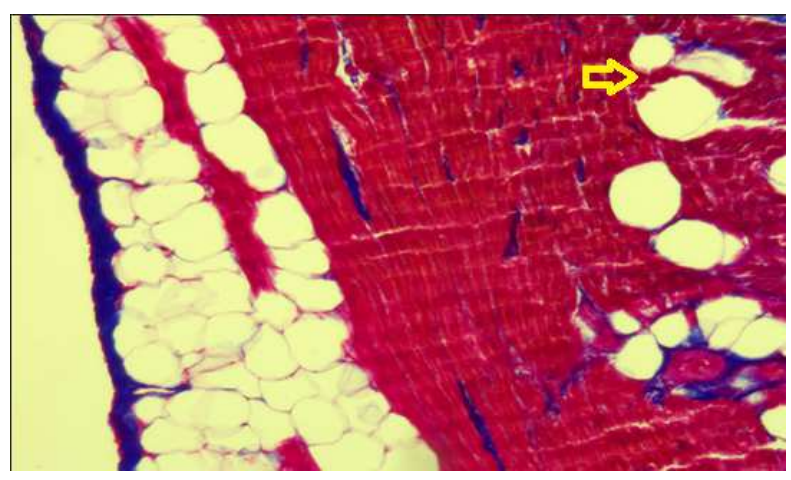

Fig. 2. CDVD group. Cardiomyocytes. Epicardium. Cardiomyocytes fatty degradation. Mallory, x200

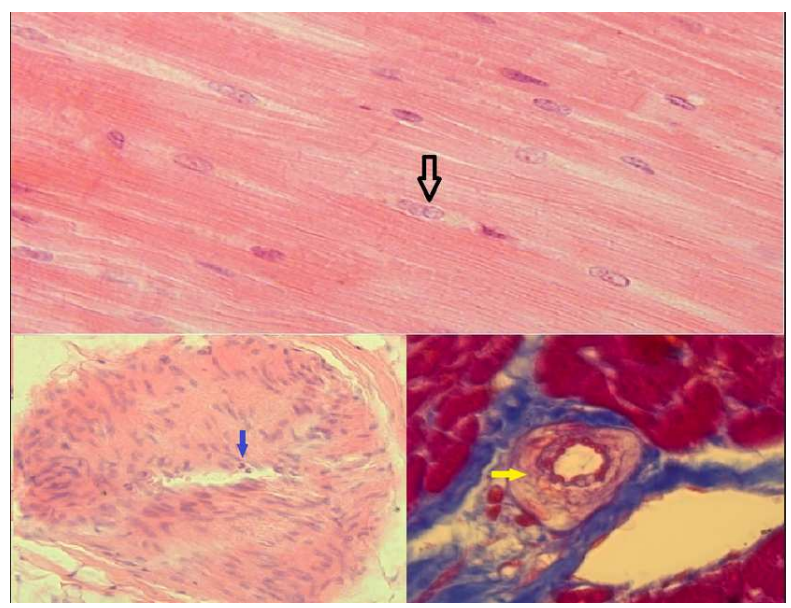

Fig. 3. Top: DoxCM. Cardiomyocytes. Two- or three nucleus cardiomyocytes (black arrow). Hematoxylin\&eosin, x400. Left bottom: DoxCM. Arteriole. Media hyperplasia. Segmented neutrophils (blue arrow). Hematoxylin\&eosin x200. Right bottom: DoxCM. Arteriole. Media hyperplasia (yellow arrow). Mallory, x 400

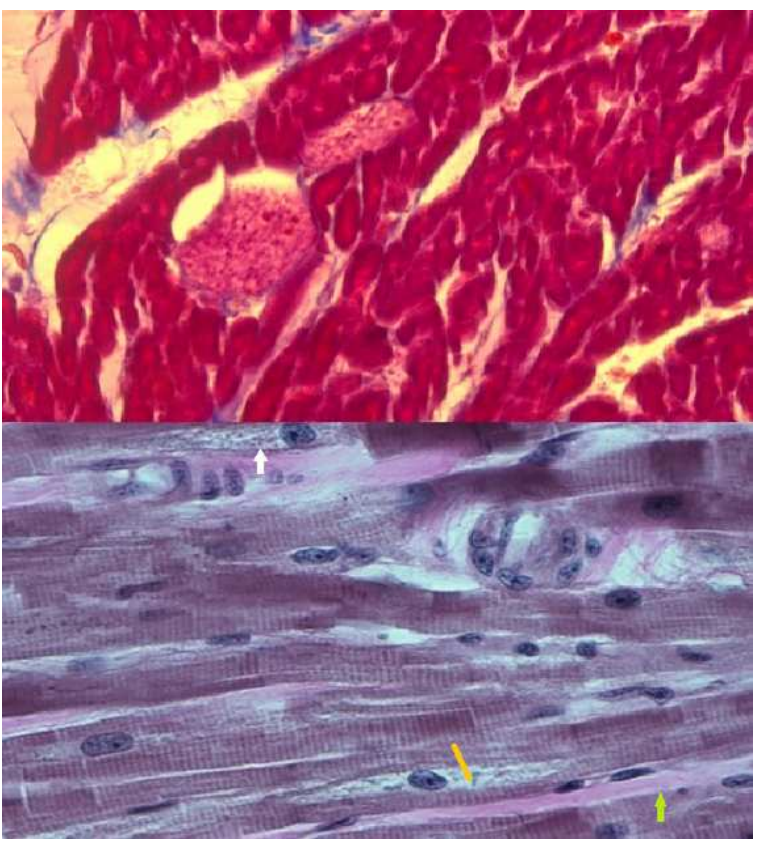

Fig. 4. Top: DoxCM. Apoptotic bodies. Mallory, x 200. Bottom: DoxCM. Separation of myofibrils. Increased amount of non-specific cytoplasm (orange stick) and lipofuscin inclusions (white arrow, granules). Connective tissue proliferation (green arrow, thin purple fiber). Metal hematoxylin\&picrofuchsin, $\mathrm{x} 400$

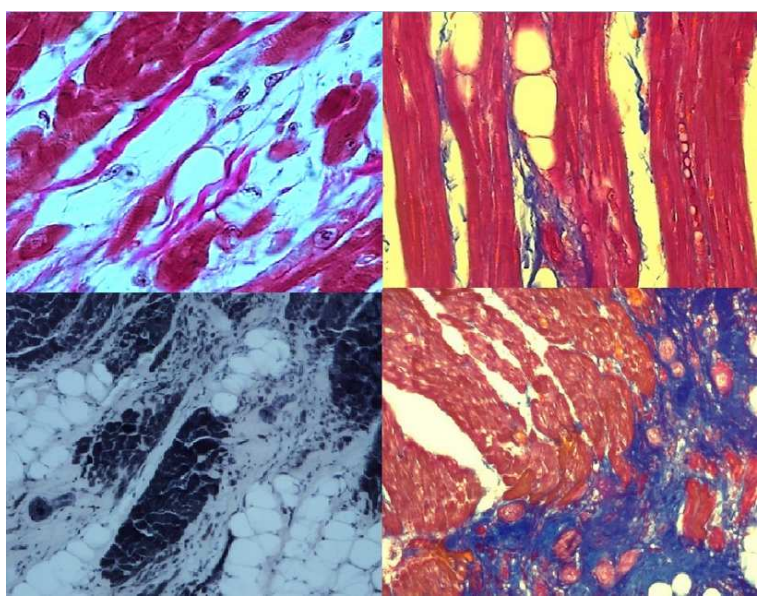

Fig. 5. Left top: DCM. Cardiomyocytes degeneration, significant fiber tissue proliferation. Hematoxylin\&eosin $x 400$. Right top: DCM. Degeneration and vacuolization of cardiomyocytes. Adipocytes, increase collagen fibers (blue). Mallory $\mathrm{x} 400$. Left bottom: Significant proliferation of connective tissue. Metal hematoxylin x100. Right bottom: DCM. Fibrosis. Mallory, x100

Final part of this study is estimation of ultramicroscopic changes in myocardium with different diseases.

In control animals, we admitted normal sarcomere morphology. Nucleuses mostly had round form or 
slightly prolonged. Chromatin located evenly in karyoplasm and more compact near internal nuclear membrane. Mitochondria are located between myofibrils and had mostly round form, with normal formation of cristae. There non-significant amounts of glycogen granules and lipid droplets (Fig. 6).

In CDVD group, we observed mostly unchanged morphology, but admitted several alterations. Intercellular spaces are not changed. Nuclear chromatin is less distributed in karyoplasm and concentrated near inner nuclear membrane. Sarcolemma had bulges with enlarged mitochondria in them. Mitochondria alterations are very variable. We observed mitochondria edema, malformation, cristae disorganization, vacuolization, petrification and formation of pseudomyelin bodies. Rarely we admitted that matrix had many small granules of medium electric density. In sarcoplasm we observed lipid droplets and phagolysosomes. Myofibrils were without significant alterations. Rarely we observed sarcoplasm full of tiny granulated substances with different electric density (partial necrosis). Endotheliocytes had no significant alterations, we observed single separated vesicles in their cytoplasm (Fig. 7).

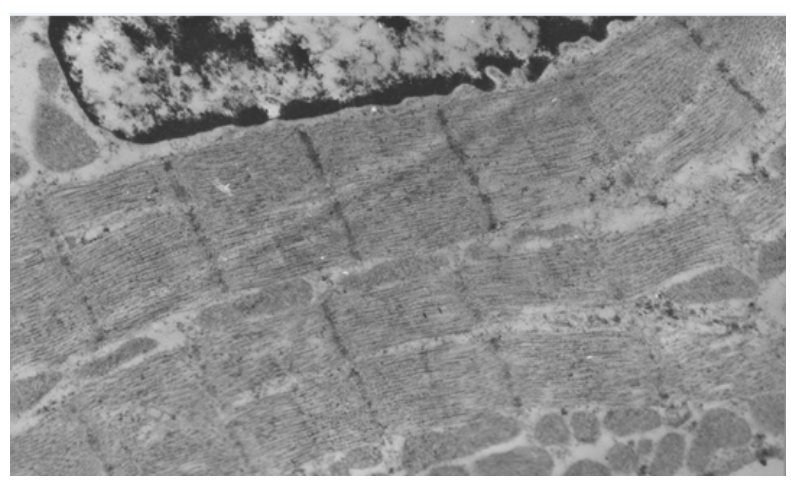

Fig. 6. Control. Right localization of myofilaments. Mitochondria are located in spaces between myofilaments. Ultramicroscopy

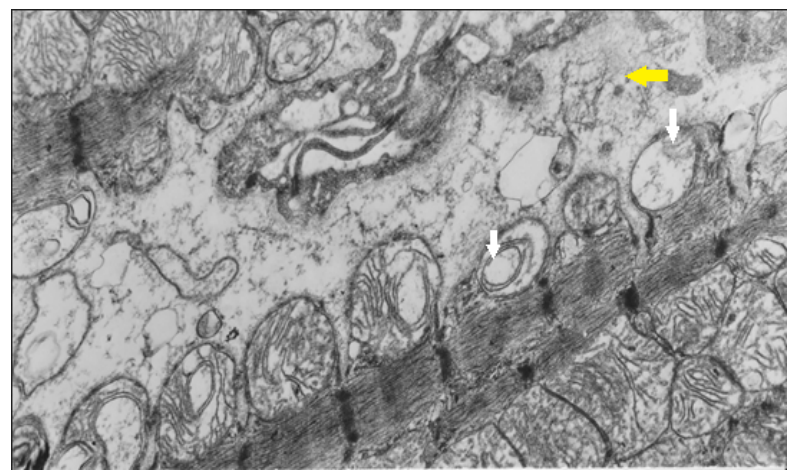

Fig. 7. CDVD. Partially degenerated mitochondria (white arrows), partial necrosis (orange arrow, non-specific cytoplasm with organelles' shadows). Ultramicroscopy
In DoxCM we admitted significant alterations of cardiomyocytes morphology. Most of cells were in edema, in some myocytes sarcolemma was separated by vacuole-like bulges. Nucleuses mostly had round form with loosely located chromatin. Mitochondria were enlarged, had different morphology and petrificated. Petrification of mitochondria was variable - we observe single and separated calcium granules and normal cristae and other cases - significant petrification. In myofibrils we observed rupture and lipid droplets, in sarcoplasm glycogen granules. Endotheliocytes cytoplasm was heavily filled with pinocytosis vesicles. Increased spaces between nucleuses, myofibrils, organelles characterized intracellular edema (Fig. 8).

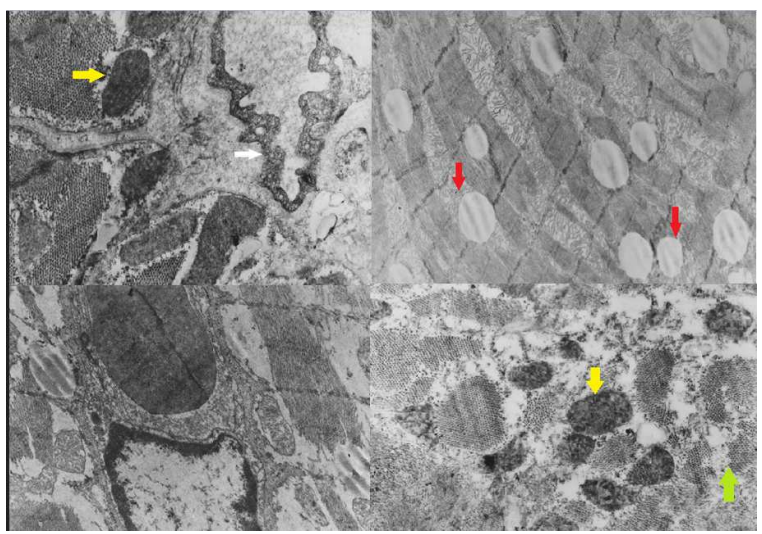

Fig. 8. Left top: DoxCM. Endotheliocyte with large multitude vesicles (white arrow). Interstitial edema. Ultramicroscopy. Right top: DoxCM. Myofibrilar separation. Lipid inclusions found right in the sarcomere (red arrows). Ultramicroscopy. Right bottom: DoxCM. Cardiomyocyte plasmolemma with vesicles and invaginations. Cardiomyocyte edema. Myofilaments destruction (green arrow). Mitochondria homogenization (yellow arrow). Ultramicroscopy. Left bottom: DoxCM. Endotheliocyte with large multitude vesicles. Cardiomyocyte edema. Myofilaments destruction. Petrification and homogenization of mitochondria. Ultramicroscopy

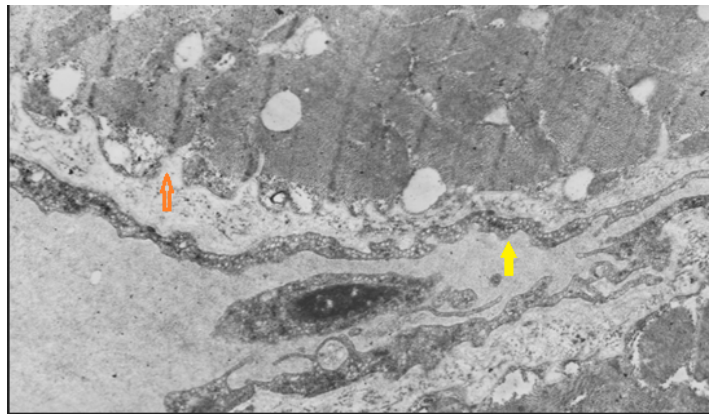

Fig. 9. DCM. Lipid drops. Interstitial and cardiomyocyte edema. Sarcolemma protrusions (red arrow). Endotheliocytecytolemma with multitude vesicles (yellow arrow). Ultramicroscopy 
In DCM group, we observed different gradations of mitochondrial alterations (edema and cristae disintegration, matrix homogenization). Most of cells had significant loss in mitochondria population. There were intermyofibrillar space increase, myofibril ruptures and high amounts of lipid droplets. In endothelial cell, we admitted many pinocytosis vesicles (Fig. 9).

\section{Discussion}

In CDVD group, we observed slightly hypertrophied cardiomyocytes, myofilaments were mostly not altered. However, we admitted adipose and connective tissue proliferation in periphery; cardiomyocytes in such sites had vacuoles, increased spaces of non-specific cytoplasm and signs of degradation. In ultramicroscopic study, we observed different stages of damage: Sites of partial necrosis, non-significant amounts of pseudomyelin bodies, but mostly morphology stayed unchanged. The most of alterations were connected with mitochondria: Edema, partial loose of cristae, rarely petrification. As a result, we can say that morphological changes in cardiomyocytes played secondary role in pathogenesis of CDVD heart failure.

In DCM group, we observed significant loss of cardiomyocytes, massive connective and adipose tissue proliferation, fibrosis. Cardiomyocytes were undergoing significant degeneration, we noticed high amounts of polynucleated cells, what characterized impaired ability to compensate and recover contractility function. Ultramicroscopic findings showed cells edema, rupture and decomposition of sarcomeres, plenty of lipid droplets and pseudomyelin bodies. Mitochondria were heavily damaged - enlarged, with matrix homogenization, petrification. This findings reflected pathogenesis of DCM-phenotype cardiomyopathy (Wilson et al., 1987).

In DoxCMgroup, we observed plenty of degrading cardiomyocytes with sarcomere separation, cytoplasm edema, polynucleation (above 4-5 in each cell), significant increase of non-specific cytoplasm, lipofuscin storage, many apoptotic bodies. Wide spread connective tissue proliferation, cardiomyocytes replaced with adipocytes - also noticed in obtained samples. As was already sad, Dox-induced damage localized specifically in subendomyocardial spaces, but also we noticed high severity of inflammatory changes in myocardial vasculature. In arterioles and venules we admitted thickened intima and media, due to proliferation of their cells and leucocytes. During ultramicroscopy study, we noticed severity of mitochondria degradation - matrix homogenization and petrification is almost in every observed sample. Sarcoplasm had many bulges and was in state of edema. Sarcomeres were ruptured and separated, between myofilaments we found plenty of lipid droplets. One of most notable findings was high activity of endothelial cells in liquid transport. High vacuolization of cytoplasm characterized increased water transport to cardiomyocytes, what could be explained by defects in sarcolemma function, rise in osmoticloncotic pressure due to severe Dox-induced damage.

\section{Conclusion}

In this study, we defined some differences in morphological and metabolic aspects between heart failure associated with different nosological units. During histological and ultramicroscopic study, we defined some specific features for each group. Myocardial remodeling is mostly located in subepicardial sites in CDVD, while in DCM and DoxCM - subendocardial sites.

Limitations in this study are connected with mixture of age, sex and breed in each group, but we should admit that in CDVD group, we had only small breeds, in DCM group - predisposed large-breed dogs, DoxCM group has mostly half-breed dogs and group of healthy dogs were a mixture of breeds. In this article we posted figures with well-expressed changes binded to characterized pathology.

\section{Acknowledgement}

Special thanks to Toropova J.V., Zelinskaya I.V. for providing an opportunity to analyze studied substrates; Korolkova E.D. for help, advices and consultations

\section{Ethics}

In all groups euthanasia were admitted in cases of endstage and refractory heart failure and inability to sustain live functions or by owners will. Obtaining of samples were agreed with owners. All experiment procedures didn't stay in conflict with regulations of European convention (Strasburg, 1985) and WSPA requirements.

\section{References}

Billingham, M.E., J.W. Mason, M.R. Bristow and J.R. Daniels, 1978. Anthracycline cardiomyopathy monitored by morphologic changes. Cancer Treat. Rep., 62: 865-872. PMID: 667860

Broder, H., R.A. Gottlieb and N.E. Lepor, 2008. Chemotherapy and cardiotoxicity. Rev. Cardiovasc. Med., 9: 75-83.

Minotti, G., P. Menna, E. Salvatorelli, G. Cairo and L. Gianni, 2004. Anthracyclines: Molecular advances and pharmacologic developments in antitumor activity and cardiotoxicity. Pharmacol. Rev., 56: 185-229. DOI: 10.1124/pr.56.2.6 
Olson, R.D. and P.S. Mushlin, 1990. Doxorubicin cardiotoxicity: Analysis of prevailing hypotheses. FASEB J., 4: 3076-3086. PMID: 2210154

Swain, S.M., F.S. Whaley and M.S. Ewer, 2003. Congestive heart failure in patients treated with doxorubicin: A retrospective analysis of three trials. Cancer, 97: 2869-2879. PMID: 12767102
Wilson, J.R., P. Douglas, W.F. Hickey, V. Lanoce and N. Ferraro et al., 1987. Experimental congestive heart failure produced by rapid ventricular pacing in the dog: Cardiac effects. Circulation, 75: 857-867. DOI: 10.1161/01.CIR.75.4.857 